\title{
A Simulation Study of Linear Coupling Effects and Their Correction in RHIC
}

\author{
G. Parzen
}

November 1992

\section{R H I C P R O J E C T}

\section{in}

$\sqrt{14} 08$

\author{
Brookhaven National Laboratory
Associated Universities, Inc. \\ Upton, NY 11973
}

Under Contract No. DE-AC02-76CH00016 with the UNITED STATES DEPARTMENT OF ENERGY 


\title{
A Simulation Study of Linear Coupling Effects and Their Correction in RHIC
}

\author{
G. Parzen \\ Accelerator Development Department \\ Brookhaven National Laboratory \\ November 1992
}

\section{Introduction}

This paper describes a possible skew quadrupole correction system for linear coupling for the RHIC92 lattice. A simulation study has been done for the correction system. Results are given for the performance of the correction system, and the required strength of the skew quadrupole correctors.

An important effect of linear coupling in RHIC is to shift the tune $\nu_{x}, \nu_{y}$, sometimes called tune splitting. Most of this tune splitting can be corrected with a two family skew quadrupole correction system. For RHIC92, the same 2 family correction system will work for all likely choices of $\beta^{*}$. This was not the case for the RHIC91 lattice where different families of correctors were needed for different $\beta^{*}$.

The tune splitting described above which is corrected with a 2 family correction system is driven primarily by the $\nu_{x}-\nu_{y}$ harmonic of the skew quadrupole field given by the field multipole $a_{1}$. There are several other effects of linear coupling present which are driven primarily by the $\nu_{x}+\nu_{y}$ harmonics of the skew quadrupole field, $a_{1}$. These include the following

1. A higher order residual tune shift that remains after correction with the 2 family correction system. This tune shift is roughly quadratic in $a_{1}$.

2. Possible large changes in the beta functions.

3. Possible increase in the beam size at injection due to the beta function distortion and the emittance distortion at injection. 
The simulation study computes the magnitude of two of these effects, the higher order residual tune shift and the change in the beta functions.

\section{The 2 Family Correction System}

The 2 family correction system is based on canceling the driving term for the nearby difference resonance, $\nu_{x}-\nu_{y}=p, p$ being an integer. For the $\nu_{x}-\nu_{y}=0$ resonance the driving term may be written as

$$
\begin{aligned}
\Delta \nu & =\frac{1}{4 \pi \rho} \int d s\left(\beta_{x} \beta_{y}\right)^{\frac{1}{2}} a_{1} \exp \left[i \bar{\nu}\left(\theta_{x}-\theta_{y}\right)\right] \\
\bar{\nu} & =\left(\nu_{x}+\nu_{y}\right) / 2 \\
\theta_{x} & =\psi_{x} / \nu_{x}, \theta_{y}=\psi_{y} / \nu_{y}
\end{aligned}
$$

One needs two families of $a_{1}$ correctors to correct both the real and imaginary parts of $\Delta \nu$. The phase of the exponent in $(2.1)$ is nearly $\psi_{x}-\psi_{y}$, so the two families should be located at places where $\psi_{x}-\psi_{y}$ differ by $\pi / 2$. One obvious place to put the $a_{1}$ correctors is near the high beta quadrupoles Q2 and Q3, where they are most effective. It will be seen from Fig. 1 that all the high beta quadrupoles have $\psi_{x}-\psi_{y} \simeq 0$, and thus another family of $a_{1}$ correctors is needed at a location where $\psi_{x}-\psi_{y} \simeq \pi / 2$.

Fig. 1 plots $\left(\psi_{x}-\psi_{y}\right) / 2 \pi$ against the path distance $s$ for a RHIC92 lattice with six $\beta^{*}=10$ insertions and also for $\operatorname{six} \beta^{*}=2$ insertions. Looking at the $\beta^{*}=10$ case, one sees that $\psi_{x}-\psi_{y} \simeq 0$ at the high beta quadrupoles Q2,Q3, while $a_{1}$ correctors near Q4 and Q5 have $\psi_{x}-\psi_{y} \simeq \pi / 2$. It is proposed that one family of $a_{1}$ correctors be located near each Q2 or Q3, which will be called the Q23 family and one family be located near each Q4 or Q5, which will be called the Q45 family. For the $\beta^{*}=2$ case, the phases are not as perfectly chosen but these two families will be adequate in this case too, although about $20 \%$ less effective. 


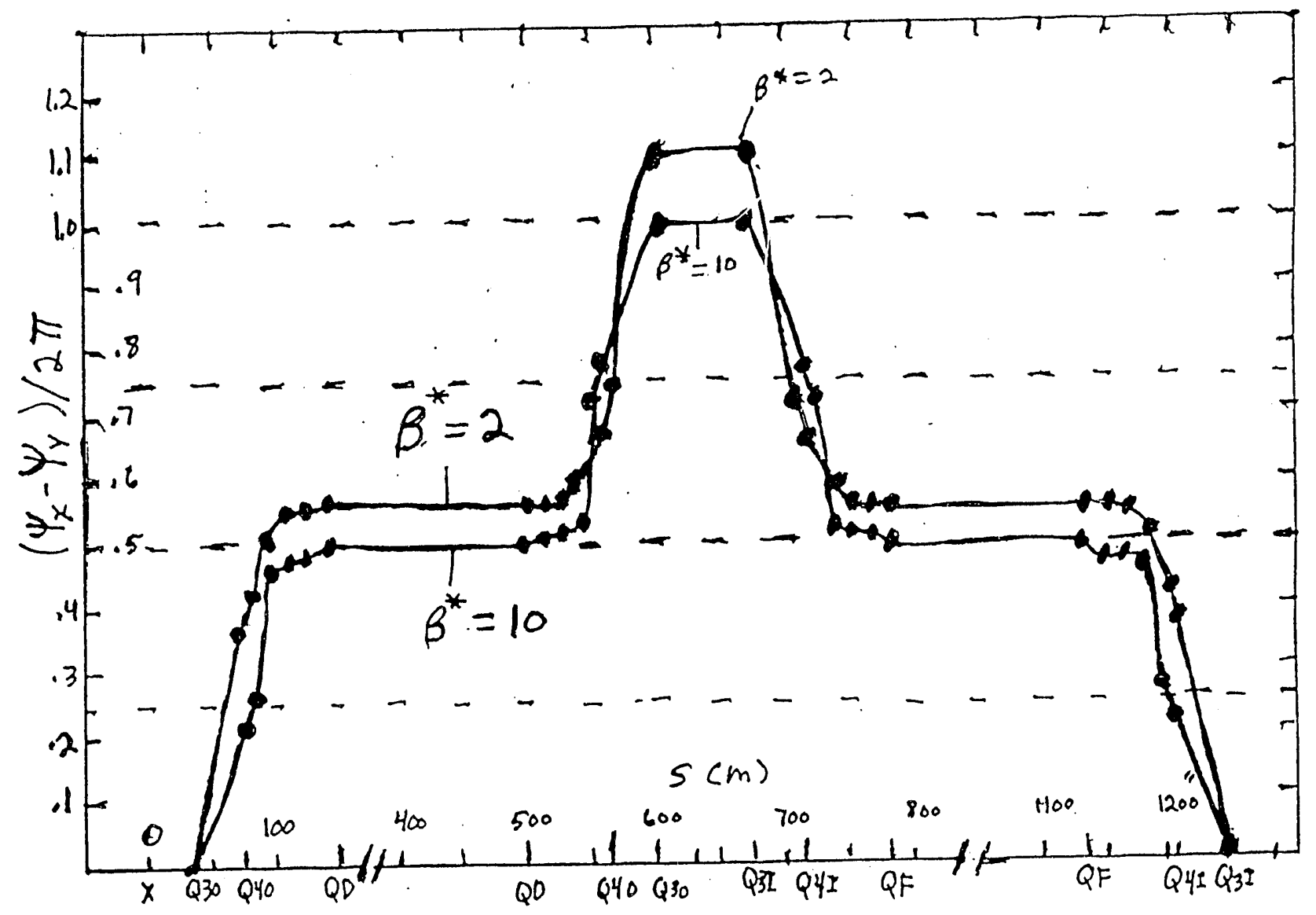

Figure 1: Plot of $\left(\psi_{x}-\psi_{y}\right) / 2 \pi$ against $s$ for the RHIC92 lattice with $6 \beta^{*}=10$ and $6 \beta^{*}=2$ insertions. Each dot on the curves indicates the location of a quadrupole.

Fig. 2 plots $\left(\psi_{x}-\psi_{y}\right) / 2 \pi$ against $s$ for $\beta^{*}=1,2,10$ and 16. From this plot, it seems likely that the proposed 2 families will work for the entire range of $\beta^{*}=1$ to $\beta^{*}=16$. The $\psi_{x}-\psi_{y}$ for $\beta^{*}=1$ nearly overlaps the curve for $\beta^{*}=2$ except for the indicated points for $\beta^{*}=1$. 


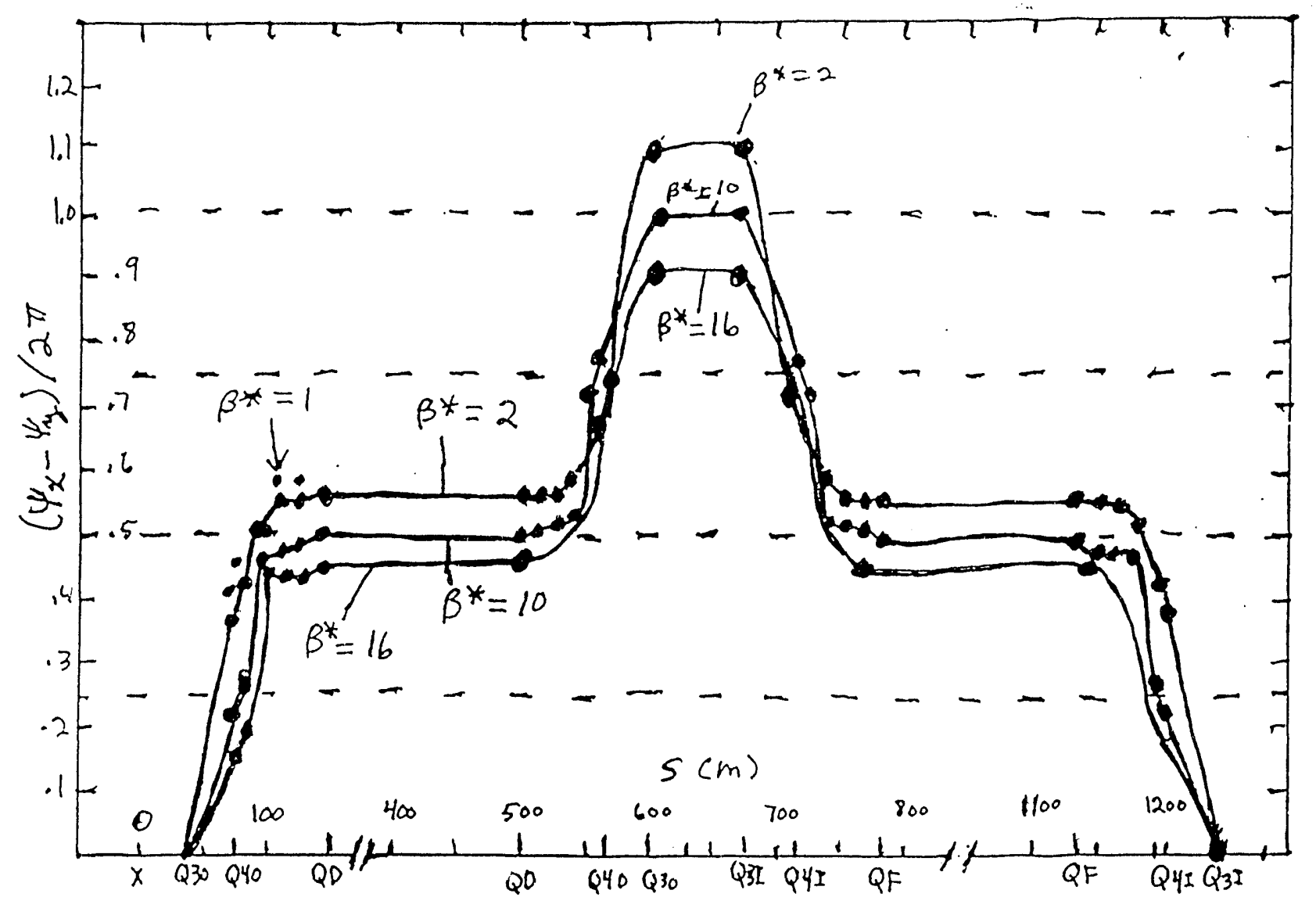

Figure 2: Plot of $\left(\psi_{x}-\psi_{y}\right) / 2 \pi$ against $s$ for RHIC92 lattices with $\beta^{*}=1,2,10,16$. The $\beta^{*}=1$ curve nearly overlaps the $\beta^{*}=2$ curve except for the indicated points for $\beta^{*}=1$. 


\section{Simulation Study Results}

For this simulation study the random $a_{1}$ and $b_{1}$ present in the magnets have a magnitude given at the end of this section. If the random $a_{1}$ and $b_{1}$ can be reduced then the effects, the tune shift and beta function shifts, can be scaled in an appropriate w.y. In this study the random $a_{1}$ and the random $b_{1}$ are both assumed to be present. However, the tune shift due to random $b_{1}$ is corrected using the QF and QD quadrupoles. Thus the tune shifts shown below are due to the random $a_{1}$ errors and the $a_{1}$ correctors. The presence of the random $b_{1}$ errors does affect the shift in the beta functions.

Table 1 gives the results for the tune, $\nu_{1}, \nu_{2}$, and the maximum beta functions at $\mathrm{QF}$ and $\mathrm{QD}, \beta_{1}, \beta_{2}$ for ten different distributions of the random $a_{1}$ and $b_{1}$. Results are shown before the 2 family correction system is applied, the uncorrected case, and after the correction is applied, the corrected case. The correctors are set to cancel the driving terms $\Delta \nu$ given by Eq. (2.1). The RHIC92 lattice has $6 \beta^{*}=2$ insertions.

Table 1. Results for the correction of the tune splitting for a RHIC92 lattice with six $\beta^{*}=2$ insertions using a 2 family correction system set to make $\Delta \nu=0$. The unperturbed tune is 28.826, 28.821. $\beta_{1}$ is the maximum $\beta_{1}$ at a $\mathrm{QF}$ and $\beta_{2}$ is the maximum $\beta_{2}$ at a $Q D$. The maximum unperturbed beta function is $50 \mathrm{~m}$.

\begin{tabular}{|c|c|c|c|c|c|c|c|c|c|c|}
\hline $\begin{array}{l}\text { Field } \\
\text { Error }\end{array}$ & $\nu_{1}$ & $-\nu_{2}$ & $\begin{array}{c}\text { uncorrected } \\
\left|\nu_{1}-\nu_{2}\right| \\
/ 10^{-3}\end{array}$ & $\begin{array}{l}\beta_{1} \\
(\mathrm{~m})\end{array}$ & $\begin{array}{l}\beta_{2} \\
(\mathrm{~m})\end{array}$ & $\nu_{1}$ & $\nu_{2}$ & $\begin{array}{c}\text { corrected } \\
\left|\nu_{1}-\nu_{2}\right| \\
/ 10^{-3}\end{array}$ & $\begin{array}{l}- \\
\beta_{1} \\
(\mathrm{~m})\end{array}$ & $\begin{array}{l}\beta_{2} \\
(\mathrm{~m})\end{array}$ \\
\hline 1 & .854 & .792 & 62 & 67 & 65 & .828 & .819 & 9 & 62 & 66 \\
\hline 2 & .860 & .791 & 69 & 78 & 69 & .829 & .822 & 7 & 63 & 58 \\
\hline 3 & .835 & .814 & 21 & 61 & 62 & .829 & .819 & 10 & 64 & 63 \\
\hline 4 & .868 & .801 & 67 & 103 & 94 & .839 & .830 & 9 & 79 & 94 \\
\hline 5 & .840 & .791 & 49 & 88 & so & .829 & .820 & 9 & 62 & 65 \\
\hline 6 & .892 & .807 & 85 & 127 & 101 & .857 & .836 & 21 & 66 & 68 \\
\hline 7 & .906 & .779 & 127 & 120 & 94 & .872 & .831 & 41 & 102 & 72 \\
\hline 8 & .941 & .709 & 232 & 92 & 105 & .832 & .817 & 15 & 65 & 59 \\
\hline 9 & & - & unstable & - & & .876 & .856 & 20 & 94 & 86 \\
\hline 10 & .782 & .870 & 88 & 69 & 75 & .834 & .821 & 13 & 63 & 72 \\
\hline
\end{tabular}

Table 1 shows that one can get tune splittings as large as $\left|\nu_{1}-\nu_{2}\right|=232 \times 10^{-3}$ before correction. One error distribution leads to unstable motion before correction, although this error distribution would not be unstable for the $\beta^{*}=10$ lattice. After correction, with the correctors set to cancel the driving term $\Delta \nu$, there remains a residual tune shift 
with the largest $\left|\nu_{1}-\nu_{2}\right|=41 \times 10^{-3}$. Previous studies ${ }^{1}$ indicate that this residual tune shift is roughly quadratic in $a_{1}$ if $\nu_{x}, \nu_{y}$ are close to the difference resonance.

Table 1 also shows large shifts for the beta functions, as much as $\Delta \beta / \beta=100 \%$ after correction. It has been shown that the beta function shift ${ }^{2}$ and the higher order residual tune shift ${ }^{3}$ are driven primarily by the harmonics of $a_{1}$ close to $\nu_{x}+\nu_{y}$. Part of the beta function shift is due to random $b_{1}$ which can cause a maximum $\Delta \beta / \beta$ of about $20 \%{ }^{4}$

Similar results have also been computed for a RHIC lattice with $6 \beta^{*}=10$ insertions. In this case the effects are smaller, and the largest residual tune splitting found is $\left|\nu_{1}-\nu_{2}\right|=$ $14 \times 10^{-3}$, and the largest beta function found at QF or QD is $\beta_{1}=61$ at a QF after correction.

In Table 1 , the 2 family correction system was set to cancel the driving term $\Delta \nu$, Eq. (2.1). In operating accelerators, the correction system is usually set to minimize the tune splitting, as $\Delta \nu$ is not known. Table 2 gives the results for $\nu_{1}, \nu_{2}$ and $\beta_{1}, \beta_{2}$ when the 2 family correction is set to minimize $\left|\nu_{1}-\nu_{2}\right|$. The lattice is a RHIC92 lattice with $6 \beta^{*}=2$ insertions. Table 2 gives the results only after correction.

Table 2. Results for the correction of the tune splitting for a RHIC92 lattice with six $\beta^{*}=2$ insertions using a 2 family correction system set to minimize $\left|\nu_{1}-\nu_{2}\right|$. Results are shown after correction.

\begin{tabular}{|cccccc|}
\hline Field Error & $\nu_{1}$ & $\nu_{2}$ & $\left|\nu_{1}-\nu_{2}\right| / 10^{-3}$ & $\beta_{1}$ & $\beta_{2}$ \\
\hline 1 & .824 & .823 & 1 & 59 & 59 \\
2 & .828 & .824 & 4 & 56 & 55 \\
3 & .827 & .821 & 6 & 54 & 55 \\
4 & .836 & .833 & 3 & 72 & 71 \\
5 & .825 & .821 & 3 & 65 & 63 \\
6 & .856 & .837 & 19 & 58 & 64 \\
7 & .856 & .838 & 18 & 64 & 63 \\
8 & .831 & .818 & 13 & 63 & 58 \\
9 & .876 & .856 & 20 & 93 & 86 \\
10 & .829 & .826 & 3 & 74 & 67 \\
\hline
\end{tabular}

In Table 2, the results for the tune splitting is somewhat smaller than found in Table 1. The largest tune splitting found after correction is $\left|\nu_{1}-\nu_{2}\right|=20 \times 10^{-3}$. The largest beta function shift after correction is $\Delta \beta / \beta=86 \%$. The results are smaller but still appreciable. 
For a RHIC92 lattice with $6 \beta^{*}=10$ insertions, and the correctors set to minimize the tune splitting, the largest residual tune splitting found is $\left|\nu_{1}-\nu_{2}\right|=8 \times 10^{-3}$ and the largest beta function at $\mathrm{QF}$ or $\mathrm{QD}$ after correction is $\beta_{1}=59$ at a $\mathrm{QF}$.

\section{Required Correction Strengths}

In the above simulation, the largest corrector strengths needed are the following

$$
\begin{aligned}
& \int d s B_{0} a_{1}=15 \mathrm{kG}, \quad \text { family Q45 } \\
& \int d s B_{0} a_{1}=6 \mathrm{kG}, \quad \text { family Q23 }
\end{aligned}
$$

\section{Random $a_{1}$ and $b_{1}$ Errors Assumed in Study}

The random quadrupole errors are due to a number of sources that include construction errors in the magnet coils, effective length errors in the quadrupoles, and rotational errors in the positioning of the quadrupoles.

$a_{1}$ and $b_{1}$ are defined so that the field due to $a_{1}$ and $b_{1}$ on the median plane is given by

$$
\begin{aligned}
& B_{y}=B_{0} b_{1} x \\
& B_{x}=B_{0} a_{1} x
\end{aligned}
$$

where $B_{0}$ is the main dipole field.

The rms random $a_{1}, b_{1}$ used in this study are given in the following table.

\begin{tabular}{lll} 
Source & $\begin{array}{l}a_{1} / 10^{-5} \\
\left(\mathrm{~cm}^{-1}\right)\end{array}$ & $\begin{array}{l}b_{1} / 10^{-5} \\
\left(\mathrm{~cm}^{-1}\right)\end{array}$ \\
\hline Dipole coil error & 16.8 & 8.4 \\
Quadrupole coil error & 15 & 15 \\
Quadrupole effective length & - & 40 \\
Quadrupole rotation error & 40 & - \\
\hline
\end{tabular}

The assumed effective length error in the quadrupoles is $\Delta L / L=2 \times 10^{-3} \mathrm{rms}$. The assumed rotational error in the quadrupoles is $\Delta \theta=1 \times 10^{-3} \mathrm{rad} \mathrm{rms}$. 


\section{References}

1. G. Parzen, Tune Shifts and Beta Function Shifts Due to Linear Coupling, Advanced Beam Dynamics Workshop, AIP Conf. Proc. No. 255 p. 131 (1991).

2. G. Parzen, Theory of the Beta Function Shift Due to Linear Coupling, BNL Report AD/RHIC-102 (1991).

3. G. Parzen, Theory of the Tune Shift Due to Linear Coupling, BNL Report AD/RHIC-100 (1991).

4. G. Parzen, Linear Random Quadrupole Effects, BNL Report AD/RHIC-AP-71 (1988). 

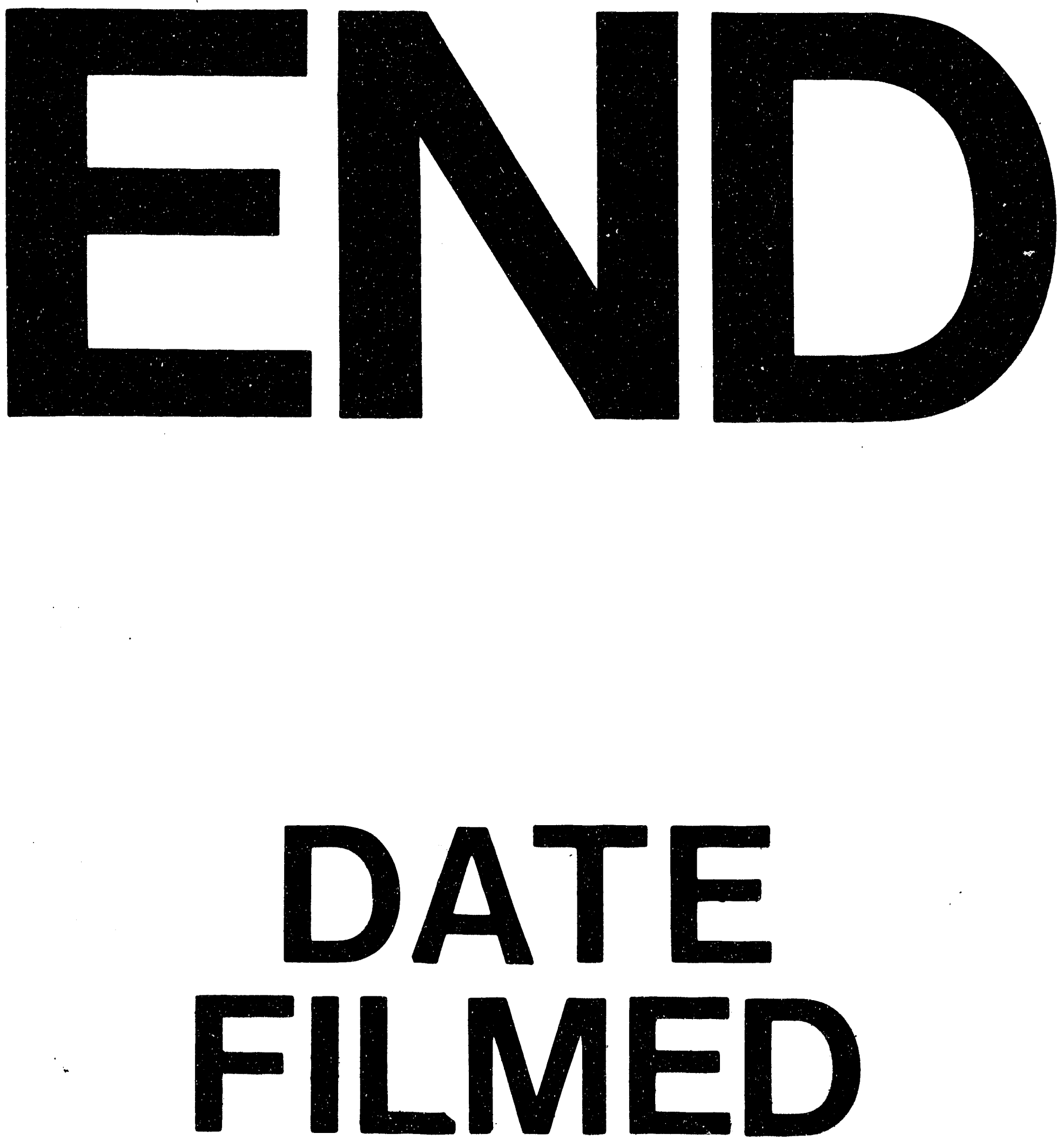

1

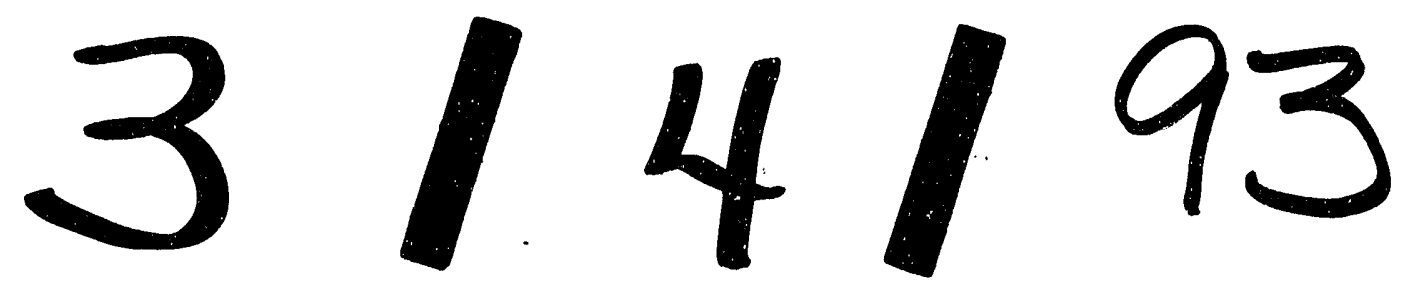


